\title{
Effective forces in colloidal mixtures: from depletion attraction to accumulation repulsion
}

\author{
A.A. Louis ${ }^{a}$, E. Allahyarov ${ }^{b}$ H. Löwen ${ }^{b}$, and R.Roth ${ }^{c}$ \\ ${ }^{a}$ Department of Chemistry, Lensfield Rd, Cambridge CB2 1EW, UK \\ ${ }^{b}$ Institut für Theoretische Physik II, Heinrich-Heine-Universität Düsseldorf, D-40225 Düsseldorf, Germany \\ ${ }^{c}$ Max-Planck Institut für Metallforschung, Heisenbergstrasse 1, D-70569 Stuttgart, Germany and ITAP, University of \\ Stuttgart, Pfaffenwaldring 57, D-70569 Stuttgart, Germany
}

(October 26, 2018)

\begin{abstract}
Computer simulations and theory are used to systematically investigate how the effective force between two big colloidal spheres in a sea of small spheres depends on the basic (big-small and small-small) interactions. The latter are modeled as hard-core pair potentials with a Yukawa tail which can be both repulsive or attractive. For a repulsive small-small interaction, the effective force follows the trends as predicted by a mapping onto an effective non-additive hard-core mixture: both a depletion attraction and an accumulation repulsion caused by small spheres adsorbing onto the big ones can be obtained depending on the sign of the big-small interaction. For repulsive bigsmall interactions, the effect of adding a small-small attraction also follows the trends predicted by the mapping. But a more subtle "repulsion through attraction" effect arises when both bigsmall and small-small attractions occur: upon increasing the strength of the small-small interaction, the effective potential becomes more repulsive. We have further tested several theoretical methods against our computer simulations: The superposition approximation works best for an added bigsmall repulsion, and breaks down for a strong big-small attraction, while density functional theory is very accurate for any big-small interaction when the small particles are pure hard-spheres. The theoretical methods perform most poorly for small-small attractions.
\end{abstract}

82.70Dd,61.20.Gy

\section{INTRODUCTION}

A fundamental description of colloidal interactions based on statistical mechanics is needed to understand and predict the stability and the phase behavior of colloidal suspensions [1.2]. Most inter-colloidal forces are effective in the sense that some microscopic degrees of freedom are averaged out. This concept of mean or effective interactions [3 5 ] is crucial to bridge the different length scales involved in colloidal systems and has been exploited in many different circumstances. Examples of the microscopic degrees of freedom include solvent particles [6], smaller colloidal particles [7], added polymer coils [8 11] or monomers of (grafted) polymer chains 12 15] as well as counter-ions [16,17] and salt ions [18] in the case of charged suspensions. The resulting effective forces turn out to exhibit a wide range of features: They can be attractive, repulsive, or oscillatory, and are an important key to understanding colloidal stability as well as flocculation and coagulation [1].

In the present paper we consider a binary colloidal mixture of big and small colloidal particles and investigate the distance-resolved effective force and potential between two big colloidal spheres surrounded by many small colloidal spheres, as depicted in Fig. 1. The small particles are averaged out so that one is left with an effective pair potential between the big ones, which is superimposed onto the direct big-big interaction. The form of these effective interactions depends sensitively on the basic big-small and small-small interactions.
Most previous studies have focused on a hard-sphere (HS)-like interaction between the colloidal particles, with a special emphasis on two cases: (1) the Asakura-Oosawa model, which was originally designed to describe mixtures of sterically-stabilized colloids and nonadsorbing polymer coils [9]. In this strongly non-additive [19] model, the big-small interaction is HS like, while the small-small interaction is ideal gas like. The effective pair potential between two colloids can be exactly calculated and has an attractive well proportional to the density of the small particles, and a range equal to their diameter. (2) Much work has also focused on the complementary model of additive HS mixtures, inspired in part by a prediction that they might phase-separate 20]. The effective depletion forces were obtained by computer simulation [21,22], approximate theories [23,24], density functional theory (DFT) [25,26], and experiments [7,27,28]. Simplified potentials were used to investigate the phasebehavior of binary HS mixtures [29], where it was found that fluid-fluid phase-separation, when it occurs [30,31, was always metastable w.r.t. fluid-solid phase-separation.

In this work we generalize these studies to arbitrary big-small and small-small interactions. Our study is motivated by the fact that real colloidal suspensions typically have soft interactions which are beyond the HS model. These soft interactions can easily be tuned, for example by changing the solvent composition, the amount of added salt, the surface charge, etc..., which provides a route to tailoring the effective interactions between the big particles. Hence it is essential to understand on a full 
statistical level how the basic big-small and small-small interactions affect the effective big-big interactions in a binary colloidal mixture.

There has been some previous work on how non-HS like interactions affect the effective big-big interactions, see e.g. refs. [3, 32 43], which showed that the differences with the pure HS case could be substantial. However, with a few exceptions, all these studies were performed with approximate methods such as integral equations. Our goal here is to provide benchmark exact computer simulation results for a set of 9 different parameters corresponding to combinations of attractive and repulsive small-small and big-small interactions. This allows us to systematically study the trends in the effective forces as induced by the basic interactions. It also allows us to test several theoretical techniques, namely DFT, the superposition approximation [44], and a mapping to a non-additive HS model [30].

Generalizing from an additive to a non-additive HS mixture already leads to a much richer class of effective potentials 45, 46, 30,31, which can be exactly calculated from an accurate theory of additive HS mixtures [26. Similarly, we find here that the effective big-big interactions are profoundly influenced by the basic big-small and small-small interactions. The trends can be summarized as follows: Adding a big-small repulsion results in enhanced attraction through the standard depletion mechanism [8,9]. Adding a big-small attraction leads to an accumulation of the small particles near each big one, which in turn results in a more repulsive big-big interaction (accumulation repulsion). For a given big-small interaction, adding a small-small repulsion also results in an enhanced small particle density near the big particles, and therefore in a more repulsive big-big interaction. Adding a small-small attraction when the big-small interaction is repulsive results in a more attractive effective interaction. All these effects can be qualitatively understood from a mapping to a non-additive HS Model. But when a small-small and a big-small attraction are combined, a repulsion through attraction effect occurs, which is not captured by DFT or by our mapping scheme.

The paper is organized as follows: In Chapter II, we discuss how to formally map a two-component mixture onto an effective one-component one, and define our target quantities. In Chapter III, we describe our model for the basic interactions. We discuss our simulation results in Chapter IV, and the results of several different theories in Chapter V. Our conclusions are stated in Chapter VI.

\section{MAPPING A BINARY MIXTURE ONTO AN EFFECTIVE ONE-COMPONENT SYSTEM}

In this section we briefly describe how to map a two-component binary mixture onto an effective onecomponent system. This statistical mechanical procedure was originally developed by McMillan and Mayer
477 to trace out the solvent in a suspension, but it can be used just as well to trace out a smaller component in a binary colloid mixture.

We begin with the Hamiltonian of our two-component mixture

$$
H=K+H_{b b}+H_{s s}+H_{b s} .
$$

Here $K$ is the total kinetic energy of the mixture and three potential energy contributions are:

$$
\begin{aligned}
& H_{b b}=\sum_{i<j}^{N_{b}} \Phi_{b b}\left(\mathbf{r}_{i}^{b}-\mathbf{r}_{j}^{b}\right), \\
& H_{s s}=\sum_{i<j}^{N_{s}} \Phi_{s s}\left(\mathbf{r}_{i}^{s}-\mathbf{r}_{j}^{s}\right), \\
& H_{b s}=\sum_{i=1}^{N_{b}} \sum_{j=1}^{N_{s}} \Phi_{b s}\left(\mathbf{r}_{i}^{b}-\mathbf{r}_{j}^{s}\right),
\end{aligned}
$$

where the $\Phi_{i j}$ denote the pairwise interaction potentials, and $\mathbf{r}_{i}^{b}$ and $\mathbf{r}_{j}^{s}$ denote the coordinates of the centers of the big and small particles respectively.

The formal procedure to reduce the two-component system to an effective one-component one, described in more detail elsewhere, see e.g. [4,5, 16, 29, 31], consists of tracing out the small particles for a fixed configuration $\left\{\mathbf{r}_{i}^{b}\right\}$ of the big particles. This is most conveniently done for a semi-grand ensemble where the small particles in the volume $V$ are kept at a fixed chemical potential $\mu_{s}$. The resulting effective one-component Hamiltonian that governs the behavior of the big particles takes the form:

$$
H_{b b}^{e f f}=H_{b b}+\Omega,
$$

where $\Omega=\Omega\left(N_{b}, z_{s}, V ;\left\{\mathbf{r}_{i}^{b}\right\}\right)$ is the grand potential of a fluid of small particles at fugacity $z_{s}=\lambda_{s}^{-3} \exp \left(\beta \mu_{s}\right)$, ( $\left.\beta=1 / k_{B} T\right)$ subjected to the external potential of the fixed configuration $\left\{\mathbf{r}_{i}^{b}\right\}$ of the big particles. This grandpotential can be further expanded as a sum of (n-body) terms [29]:

$$
\Omega=\sum_{n=0}^{N_{b}} \Omega_{n}
$$

The first term, $\Omega_{0}$, is the so-called volume term, which only depends on properties of the small-particles (i.e. $\left.\Phi_{s s}\right)$ and is therefore independent of the configuration $\left\{\mathbf{r}_{i}^{b}\right\}$. The one-body term $\Omega_{1}$ can be related to $N_{b}$ times the free-energy gained by inserting a single big particle into a sea of small particles. It depends on both $\Phi_{s s}$ and $\Phi_{b s}$, but is independent of $\Phi_{b b}$. Of most interest to us here is the two-body term $\Omega_{2}$, which can be written as:

$$
\Omega_{2}\left(N_{b}, z_{s},\left\{\mathbf{r}_{i}^{b}\right\}\right)=\sum_{i<j}^{N_{b}} V_{b b}^{e f f}\left(\left|\mathbf{r}_{i}-\mathbf{r}_{j}\right|\right)
$$


where the two-body effective pair-potential $V_{b b}^{e f f}(r)$ is related to the free-energy difference between two big spheres a distance $r$ apart, immersed in a sea of small spheres at fugacity $z_{s}$, and the same system with the two spheres at $r=\infty$. It is important to notice that $V_{b b}^{e f f}(r)$ itself only depends on $\Phi_{b s}$ and $\Phi_{s s}$, and not directly on $\Phi_{b b}$. This observation was a key to understanding why the effective potentials for non-additive HS mixtures can be calculated by a theory for additive HS mixtures [30, 31, 46]. Nevertheless, through Eq. (3), the effect of $\Phi_{b b}(r)$ is felt in that it sets the range where $V_{b b}^{e f f}(r)$ is relevant.

In a similar way, higher order interactions can be derived [15], but for this scheme to remain tractable, one usually truncates at the pair level. As long as the ranges of $\Phi_{s s}$ and $\Phi_{b s}$ are small compared to the range of $\Phi_{b b}$, higher order terms are not expected to be very important [29, 31.

Thus far we have developed this formal tracing out in the semi-grand ensemble, which helps emphasize that in a mixture with many large particles, the correct effective potential $V_{b b}^{e f f}(r)$ to be used in Eq. (5) is the one fixed by the chemical potential or fugacity instead of the overall density of the smaller particles, as first emphasized by Lekkerkerker et al. [10]. It is important to keep this in mind when studying phase-behavior. However, for a practical calculation of the pair term, with only two big particles in an infinite sea of small ones, one can just as easily work in the canonical ensemble, keeping in mind that when using the obtained $V_{b b}^{e f f}(r)$ for a system with many big particles, the input density $\rho_{s}$ is really that of a reservoir of small particles kept at the same chemical potential as the full mixture of big and small particles 10,29,31.

For the case of two big-spheres a distance $r$ apart, in a bath of small particles at density $\rho_{s}$, the average force is related to the effective potential by:

$$
\mathbf{F}_{b b}^{e f f}(r)=-\frac{\partial}{\partial \mathbf{r}} V_{b b}^{e f f}(r) .
$$

This average force provides an intuitive way of understanding the effective interactions which parallels the more formal derivation above. First we define a one-body density as follows:

$$
\rho_{s}^{(1)}\left(\mathbf{r}^{\prime}\right)=<<\sum_{i}^{N_{s}} \delta\left(\mathbf{r}^{\prime}-\mathbf{r}_{\mathbf{i}}^{\mathbf{s}}\right)>>
$$

where $<<>>$ denotes a canonical thermodynamic average over the small particles. If one big particle is fixed at the origin, and one is fixed at $\mathbf{r}$, then we can define a one-particle density of the small particles $\rho_{s}^{(1)}\left(\mathbf{r}^{\prime} ; \mathbf{0}, \mathbf{r}\right)$ for that fixed configuration. The effective force induced between two big spheres by the smaller particles can then be written as 44, 21]:

$$
\mathbf{F}_{b b}^{e f f}(\mathbf{r})=-\int \rho_{s}^{(1)}\left(\mathbf{r}^{\prime} ; \mathbf{0}, \mathbf{r}\right) \frac{\partial}{\partial \mathbf{r}^{\prime}} \phi_{b s}\left(r^{\prime}\right) d \mathbf{r}^{\prime}
$$

which has the obvious physical interpretation that the total effective or mean force is simply the average of the sum over all the big-small interactions.

In the next sections, we will concentrate on the effect of changing $\Phi_{s s}(r)$ and $\Phi_{b s}(r)$, at fixed reservoir density $\rho_{s}$, on the effective interactions $F_{b b}^{e f f}(r)$ and $V_{b b}^{e f f}(r)$.

\section{MODELS}

\section{A. Interactions}

We model the basic small-small and big-small interactions defined in Eq. (2) as hard-core Yukawa pair potentials:

$$
\Phi_{i j}(r)=\left\{\begin{array}{lr}
\infty & r<\sigma_{i j} \\
\phi_{i j}(r) & \text { otherwise }
\end{array}\right.
$$

where in each case $r$ denotes the distance between the centers of the relevant particles. Throughout, we take $\phi_{b b}(r)=0$, while the big-small interaction added to the HS repulsion is given by:

$$
\phi_{b s}(r)=\frac{\epsilon_{b s} \sigma_{b s}}{r} \exp \left[-\kappa_{b s}\left(r-\sigma_{b s}\right)\right],
$$

and the small-small interaction added to the HS repulsion is given by:

$$
\phi_{s s}(r)=\frac{\epsilon_{s s} \sigma_{s s}}{r} \exp \left[-\kappa_{s s}\left(r-\sigma_{s s}\right)\right],
$$

where $\sigma_{b s}=\frac{1}{2}\left(\sigma_{b b}+\sigma_{s s}\right)$.

The Yukawa tail can be either repulsive or attractive. This gives us the possibility to change the interaction over a broad range from pure HS (which serve as a reference case) to soft repulsions and attractive tails. We note that a hard-core Yukawa interaction has been frequently used in theoretical studies on liquids, see e.g. 49] and refs therein. Both an attractive and repulsive tail is realized in various colloidal solutions: a short-ranged attractive Yukawa tail has been shown to satisfactorily model the "stickiness" in the interaction between globular protein solutions 50. A repulsive Yukawa tail, on the other hand, describes charged suspensions [2,51] where the range is controlled by the added salt concentration and the amplitude by the colloidal charge.

\section{B. Parameter combinations}

Our aim is to systematically investigate the effect of the basic big-small and small-small interactions on the effective big-big interactions. We vary the big-small interaction by changing $\beta \epsilon_{b s}$, and vary the small-small interaction by changing $\beta \epsilon_{s s}$. The other parameters are 
fixed as follows: $\sigma_{s s}=0.2 \sigma_{b b}, \kappa_{b s}=6 / \sigma_{b b}=1.2 / \sigma_{s s}$, and $\kappa_{s s}=15 / \sigma_{b b}=3 / \sigma_{s s}$. The range of the big-small interaction is of the order of the small particle size, while the range of the small-small interactions is significantly less than the small particle size. For all simulations the packing fraction of the small particles in the bulk is set to $\eta_{s}=\pi \rho_{s} \sigma_{s s}^{3} / 6=0.1$.

In total, we studied 9 different parameter combinations corresponding to taking $\beta \epsilon_{b s}$ and $\beta \epsilon_{s s}$ to be positive, negative, or zero. The detailed values are given in table 1 .

\section{COMPUTER SIMULATIONS OF EFFECTIVE FORCES AND INTERACTIONS}

\section{A. Simulation method}

Our simulation set-up contains two big spherical particles in a large cubic simulation box of length $L$ with periodic boundary conditions in all three directions. The big particles with their center-to-center separation $r$ are fixed along the body diagonal of the cubic box and $N_{s}=10000$ small mobile particles of diameter $\sigma_{s s}$ are added to the box. The box length $L=7.49 \sigma_{s s}$ is sufficiently large to exclude any spurious periodic image effects. We also studied a single big particle in the simulation box to access the one-body small-particle density $\rho_{s}(r)$ around an isolated big sphere. For two big spheres $\rho_{s}=N_{s} /\left(L^{3}-\frac{1}{3} \pi \sigma_{b b}^{3}\right)$, while for one big sphere $\rho_{s}=N_{s} /\left(L^{3}-\frac{1}{6} \pi \sigma_{b b}^{3}\right)$. We checked the asymptotic density profiles to confirm that the bulk densities were always the same.

We use a molecular dynamics (MD) code combining the velocity Verlet algorithm [52] with discrete collisions and reflections induced by the hard core of the interaction potentials in order to calculate the trajectories of the small particles. The system was carefully equilibrated and then statistical averages were computed such as the mean force acting on the big particles (see Eq. (8)) or the inhomogeneous density field $\rho_{s}(r)$ of the small particles around a single big particle. Details of our simulation procedure are described in references [18,53.

\section{B. One body density profiles}

Before we discuss the depletion potentials it is useful to first examine the density profiles $\rho_{s}(r)$ of the small particles around a single sphere.

\section{No extra big-small interaction: runs 1-3}

In Fig. 2, these are shown for runs $1-3$, where $\epsilon_{b s}=0$. Compared to the density profile of the pure HS, there is a significantly increased accumulation near the big spheres when a small-small repulsive interaction is added. To first order this can be understood by mapping the $\phi_{s s}(r)$ onto an effective HS diameter $\sigma_{s s}^{e f f}$ [54]: Adding a smallsmall repulsive interaction increases the effective HS size, and therefore also the effective HS packing fraction, resulting in a more pronounced accumulation of density near the surface. Adding a small-small attraction has the opposite effect. This is because the bulk is now more favorable for the small particles, and they are attracted to it.

\section{Added big-small repulsion: runs 4-6}

In Fig. 3, the density profiles are shown for runs $4-6$, where $\beta \epsilon_{b s}=0.82$. As expected, the repulsive big-small interaction leads to a reduction of the density at contact. Again, adding a small-small repulsion increases the density at contact, and adding a small-small attraction leads to a further depletion of the density at contact.

\section{Added big-small attraction: runs 7-9}

In Fig. 1, the density profiles are compared for runs $7-9$, where $\beta \epsilon_{b s}=-0.82$. As expected, the attractive big-small interaction results in an enhanced density at contact. Similarly to the previous two cases where $\epsilon_{b s}=0$ and $\epsilon_{b s}>0$, respectively, adding a small-small repulsion enhances the contact density w.r.t. the case of no small-small repulsion. But in contrast to the two previous cases, where adding a small-small attraction resulted in a depleted density profile w.r.t. the pure HS case, here adding $\epsilon_{s s}<0$ results in an enhanced total accumulation of the small particles near the big one. Although the contact value is slightly smaller than the case for pure HS, there is a marked accumulation further out, corresponding to a second layer of particles. The relative adsorption of the small particles around the big one is thus considerably larger than for the case of no small-small attractions. This can be understood by the following simple argument: the big-small attraction leads to an accumulation of the small particles near the surface of the big sphere. When this accumulation is large enough, it becomes favorable for the small particles to leave the bulk, and approach the surface of the big particle, where their local density is larger. Thus the two attractions amplify each other in a non-linear fashion.

In conclusion then, when comparing Figs. 2, 3 and 4, it is clear that for the parameters we have chosen, the big-small interaction has the largest relative effect on the density profiles. The effect of adding a small-small repulsion can be qualitatively understood by the larger effective sphere size and concomitant larger packing fraction. The effect of adding a small-small attraction can be qualitatively understood by the fact that the bulk is usually preferred over the surface of the particle, except when the big-small interaction is strong enough to provoke a 
non-linear enhancement of the density of small particles near a big one.

Having investigated the effect of the interactions on the one-body density profiles, we now turn to the related two-body depletion potentials.

\section{Effective pair forces and potentials}

In Fig. 5 we compare the effective depletion force between two big spheres for all nine parameter combinations detailed in table If. In Fig. 6 we compare the related depletion potentials. First we note that changing the big-small interaction is the dominant effect: the depletion forces and potentials split naturally into three groups: no added big-small interaction (solid lines, runs $1-3$ ), big-small attraction (dotted lines, runs 4-6), and big-small repulsion (dot-dashed lines, runs $7-9$ ). We will treat each case in turn.

\section{No extra big-small interaction: runs 1-3}

As was already seen for the one-body profiles, adding a small-small repulsion (run 2) results in a larger effective small-sphere size and packing fraction, which is reflected in more pronounced oscillations compared to the pure HS case (run 1). These are evident both in the effective force and in the effective pair potential. Adding a small-small attraction (run 3) results in a reduced density near a big particle, as seen in Fig. 2. This would imply that each big sphere excludes slightly more free volume than if there were no small-small attractions, which, in turn, implies a slightly more attractive potential, as is observed.

\section{Added big-small repulsion: runs 4-6}

The dominant effect of adding a big-small repulsion is to make the effective forces and potentials much more attractive. This can be understood with the classical picture of depletion [8.9]: Adding a big-small repulsion results in a larger depletion layer around each large particle. When two large spheres approach, the amount of doubly excluded volume is therefore larger, resulting in a more attractive effective (depletion) potential or force. Again, adding a small-small repulsion (run 5) results in enhanced layering as compared to the pure HS small particles. Adding a small-small attraction (run 6) has only a weak effect similar to what was seen for run 3 .

\section{Added big-small attraction: runs 7-9}

The dominant effect of adding a big-small attraction is to make the effective forces and potentials much more repulsive. This can be qualitatively understood from the fact that the big-small attraction results in an enhanced density of small particles near a single large sphere. When two such large spheres approach one another, the layers of small particles around each one begin to interact, leading to an enhanced repulsion between them. As demonstrated in Fig. 4 for a given big-small attraction, adding small-small repulsion or attraction both result in a further enhanced density $\rho_{s}(r)$ of the small particles around a single large one, which in turn explains why the effective pair potentials are more repulsive for both $\epsilon_{s s}>0$ and $\epsilon_{s s}<0$. Relatively speaking, adding smallsmall attractions has the largest effect on the effective potentials, which is due to the non-linear coupling between the big-small and the small-small attractions.

\section{THEORETICAL DESCRIPTIONS OF THE EFFECTIVE FORCES AND POTENTIALS}

\section{A. Superposition approximation}

To calculate the effective forces via Eq. (8), one needs a prescription for calculating the one-body density of the small particles. In section IV, we essentially did this by computer simulations. In this section we approximate the full one-body density by a superposition of the one-body density $\rho_{s}(r)$ around an isolated single sphere [44]:

$$
\rho_{s}^{(1)}\left(\mathbf{r}^{\prime} ; \mathbf{0}, \mathbf{r}\right)=\rho_{s}\left(r^{\prime}\right) \rho_{s}\left(\left|\mathbf{r}-\mathbf{r}^{\prime}\right|\right) / \rho_{s},
$$

an approach similar in spirit to the Kirkwood superposition approximation 54]. Since all input information comes from the (radially symmetric) problem of a single sphere, this superposition approximation greatly simplifies the calculation of the two-body depletion forces. The input $\rho_{s}(r)$ could come from density functional theory or integral equation theory, as was done previously by other authors [21,34, but here we will use the $\rho_{s}(r)$ generated by our simulations and depicted in Figs. 24. The results are shown in Figs. 7 15, where we compare in detail the radial force $F_{b b}^{e f f}(r)=\mathbf{F}_{b b}^{e f f}(r) \cdot \mathbf{r} / r$ and the effective potential $V_{b b}^{e f f}(r)$ calculated with the superposition approximation, to the results obtained by direct simulations.

Figure 7 (run 1) shows the HS reference case. Here the superposition approximation works quite well. The packing fraction of the small spheres $\eta_{s}=0.1$ is rather low, so we expect that the small-small correlation effects are not very strong. The total one-body density is therefore well approximated by Eq. (12). As demonstrated by other authors 21, 34 for the pure HS case, this superposition approximation begins to break down as $\eta_{s}$ increases and two-body correlation effects become more important. For example, they found that for large $\eta_{s}$, the superposition approximation leads to an overestimate of the strength of the attractive force at contact. This is exactly what is seen in Fig. \& (run 2), where the effect of an added repulsive $\phi_{s s}(r)$ can be understood in terms of a larger effective packing fraction $\eta_{s}$. Figure 9 (run 3) 
shows that for $\epsilon_{s s}<0$ the superposition approximation shows a similar error to what was seen for run 2 , i.e. the forces and potentials are too attractive.

In Figs. $10-12$ (runs 4-6), where the repulsive $\epsilon_{b s}(r)$ induces much more attractive effective interactions than for the pure HS case, the superposition approximation is seen to work quite well for $\epsilon_{s s}=0$ and $\epsilon_{s s}>0$. This is most likely because the effective (depletion) force or potential is dominated by $\phi_{b s}(r)$, which also induces a lower $\rho_{s}(r)$ (see Fig. 3), so that small-small correlations only play a relatively minor role and Eq. (12) is rather accurate. Even for $\epsilon_{s s}<0$, the results are quite good, although the superposition approximation tends to predict forces and potentials that are too attractive, just as was found for $\phi_{b s}(r)=0$.

In Figs. 13 15 (runs 7-9), where the attractive $\phi_{b s}(r)$ induces an increased local accumulation of the small spheres near each big sphere, the superposition approximation performs less well. This is perhaps not surprising, as the enhanced density of small particles near each big sphere results in a more important role for small-small correlations, which are not well treated by the superposition approximation.

In summary then, for HS or other repulsive small-small interactions, the superposition approximation works best for low (effective) packing fractions $\eta_{s}$, where correlations between the small particles do not strongly alter the full one-body density $\rho_{s}^{(1)}\left(\mathbf{r}^{\prime} ; \mathbf{0}, \mathbf{r}\right)$ from the superposition of the densities around an isolated big sphere. The case of a strongly repulsive $\phi_{b s}(r)$, which lowers the effective packing fraction near the big spheres, is particularly well described by the superposition approximation. In contrast, the case of an attractive $\phi_{b s}(r)$, which results in an increased accumulation of small particles around each big sphere, is not as well described.

\section{B. Density Functional Theory}

In a recent development, density functional theory (DFT) has been used to derive effective potentials for additive 25.26, and non-additive 30,31,46] HS mixtures. For the additive case, quantitative agreement with computer simulations was achieved 25,26]. Since the calculations for the non-additive case were shown to be equivalent to the additive ones [30,31, a similar quantitative accuracy could be expected there. In brief, the method is based on the following exact relationship between the effective potential and the one-body direct correlation function [25,26]:

$$
\beta V_{b b}^{e f f}(\mathbf{r})=\lim _{\mu_{b} \rightarrow-\infty}\left(c_{b}^{(1)}(\infty)-c_{b}^{(1)}(\mathbf{r})\right)
$$

where $c_{b}^{(1)}(\mathbf{r})$ is defined for the case where a big sphere is fixed at the origin, and exerts a field on the small spheres and on a big test particle inserted at $\mathbf{r}$ 26]. DFT can therefore provide a route to the effective potentials since

$$
c_{b}^{(1)}(\mathbf{r})=-\beta \frac{\delta \mathcal{F}_{e x}\left[\rho_{b}, \rho_{s}\right]}{\delta \rho_{b}(\mathbf{r})}
$$

where $\mathcal{F}_{e x}\left[\rho_{b}, \rho_{s}\right]$ is the excess (over ideal) intrinsic Helmholtz free energy functional of the mixture [55]. Given some mixture functional, one can obtain the effective potentials and forces from a radially symmetric calculation of $c_{b}^{(1)}(\mathbf{r})$ in the limit of vanishing density of the big spheres. This is much simpler than say trying to use DFT to directly calculate the cylindrically symmetric one-body density $\rho_{s}^{(1)}\left(\mathbf{r}^{\prime} ; \mathbf{0}, \mathbf{r}\right)$, for use in Eq. (8) [36]. All one now needs is some prescription for the mixture functional. Here we use Rosenfeld's very successful fundamental measure theory [56], in its form valid for $\mathrm{HS}$ mixtures. As found previously 26], the DFT and simulation results for effective interactions of the pure HS case depicted in Fig. 17 (run 1) agree very well.

This DFT method can easily be extended to an arbitrary $\phi_{b s}(r)$, since this simply corresponds to an additional external field in Eq. (13). Previous DFT calculations 30 found good agreement with earlier simulations with an attractive $\phi_{b s}(r)$ 35. Here we also find very good agreement with the runs 4 , and 7 , (Figs. 10 and 13) which correspond to a finite $\phi_{b s}(r)$ but no additional $\phi_{s s}(r)$.

Since there is at present no successful two-component DFT for mixtures where the small-particles are not HS like, some approximations must be made. We chose to map the $\phi_{s s}(r)$ onto effective HS diameters $\sigma_{i j}^{\text {eff }}$ using the Barker-Henderson approach [57:

$$
\sigma_{i j}^{e f f}=\sigma_{i j}+\int_{\sigma_{i j}}^{\infty}\left(1-\exp \left[-\beta \phi_{i j}(r)\right]\right) d r
$$

where $\sigma_{i j}$ is the bare HS diameter. We then calculated effective pair potentials and effective pair forces with our full DFT approach, including an explicit $\phi_{b s}(r)$ when needed, but with the small-spheres mapped onto the effective diameters $\sigma_{s s}^{e f f}=1.447 \sigma_{s s}$ and $\sigma_{s s}^{e f f}=0.658 \sigma_{s s}$ for the repulsive and attractive $\phi_{s s}(r)$ respectively. Similarly the effective packing fraction becomes $\eta_{s s}^{\text {eff }}=0.303$ for the repulsive and $\eta_{s s}^{\text {eff }}=0.0290$ for the attractive interaction.

Firstly, for runs 2, 5, and 8 (Figs. 8, 11, and 14, respectively), which all correspond to a repulsive $\phi_{s s}(r)$, we find good agreement for no additional $\phi_{b s}(r)$ (see Fig. 8), but less good agreement for a repulsive or attractive $\phi_{b s}(r)$ (see Fig. 11 and Fig. 14 respectively).

Because of the strength of the small-small attraction, the mapping results in a very low effective packing fraction of the small particles. Overall, the DFT underestimates the effective forces for $\epsilon_{b s}=0$ and $\epsilon_{b s}>0$, as can be seen in Figs. 9 and 12. It performs rather poorly for run 9 , where the attractive $\phi_{b s}(r)$ results in a non-linear enhancement of the small-particle density profile, an effect not taken into account with our HS mapping. This suggests that a different two-component DFT, which explicitly takes into account the small-small 
attraction $\phi_{s s}(r)$ needs to be developed before we can use this route to derive accurate effective pair forces and potentials.

\section{Mapping to non-additive HS system}

In a previous paper 30] two of us proposed that the effects of big-small and small-small interactions on the $V_{b b}^{e f f}(r)$ could be understood by mapping onto those of non-additive HS systems. These have the advantage that they can be determined by an exact mapping onto the depletion potentials of additive HS mixtures [30,31, which, in turn, are well understood and for which a good parameterization exists [26]. Even a small non-additivity was shown to have a large effect on the depletion potentials.

By mapping the big-small and small-small interactions onto effective HS diameters through Eq. (15) one can define the non-additivity in terms of the parameter $\Delta$ :

$$
\sigma_{b s}^{e f f}=\frac{1}{2}\left(\sigma_{b b}+\sigma_{s s}^{e f f}\right)(1+\Delta) .
$$

Four different ways of adding interactions to introduce non-additivity were studied [30]:

(i) repulsive $\beta \phi_{s s}(r): \sigma_{b s}^{e f f}=\sigma_{b s} ; \sigma_{s s}^{e f f}>\sigma_{s s} ; \Delta<0$

(ii) attractive $\beta \phi_{s s}(r): \sigma_{b s}^{\text {eff }}=\sigma_{b s} ; \sigma_{s s}^{e f f}<\sigma_{s s} ; \Delta>0$

(iii) repulsive $\beta \phi_{b s}(r): \sigma_{b s}^{\text {eff }}>\sigma_{b s} ; \sigma_{s s}^{\text {eff }}=\sigma_{s s} ; \Delta>0$

(iv) attractive $\beta \phi_{b s}(r): \sigma_{b s}^{e f f}<\sigma_{b s} ; \sigma_{s s}^{e f f}=\sigma_{s s} ; \Delta<0$.

For details of how each of the different cases of nonadditivity affect the depletion potentials, we refer to refs. 30,31, 46 .

An example where the mapping to non-additivity works well is given in Fig. 8 (run 2), where we mapped the small-small repulsion onto an effective HS diameter (case (i)), so that the DFT result is really that of a nonadditive HS mixture with $\Delta=-0.069$. In ref. [30] we also found semi-quantitative agreement with the mapping for weak and short-ranged $\beta \phi_{b s}(r)$. In the present case, where $\beta \phi_{b s}(r)$ is stronger and longer ranged, the agreement is no longer quantitative. Nevertheless, for runs 1-8 the mapping scheme provides a qualitative explanation of the trends. I.e. for a fixed $\phi_{b s}(r)$, adding a repulsive (case (i)) or attractive (case (ii)) $\phi_{s s}(r)$ causes $V_{b b}(r)$ to become more repulsive or attractive, respectively. Similarly, for a $\phi_{s s}(r)$, adding a repulsive (case (iii)) or attractive (case (iv) $) \phi_{b s}(r)$ results in a more attractive or repulsive $V_{b b}(r)$, respectively. The only case where this scheme breaks down is run 9 , where adding $\phi_{s s}(r)<0$ for an attractive $\phi_{b s}(r)$ should fall under case (ii). But instead of inducing more attraction, the effective big-big potential becomes more repulsive. Of course this is not surprising, since the DFT results already showed that a mapping scheme misses the non-linear enhancement of the small particle density profiles.

In conclusion then, the mapping to non-additivity works best for a repulsive small-small interaction. For repulsive and attractive big-small interactions, the mapping is only qualitative. The much better semiquantitative agreement found in ref. [30] can be traced to the much weaker effect of the $\phi_{b s}(r)$ used there. Just as was seen for the direct DFT methods, it is the case of small-small attraction combined with big-small attraction which seems most difficult to capture within our mapping scheme.

\section{CONCLUSIONS}

In conclusion, we have shown how the basic interactions in a colloidal mixture control the resulting effective interactions between the big particles. This knowledge may be exploited to stabilize colloidal particles against coagulation and to tailor the colloidal phase diagrams.

Adding a repulsive $\phi_{b s}(r)$ results in a strongly enhanced attraction through the standard depletion mechanism. We also found at least two ways to obtain significantly more repulsive effective interactions caused by accumulation of the small particles (accumulation repulsion): (1) Adding repulsions between the small particles results in an enhanced accumulation near the surface of the large particles; when two large particles approach each other, this results in an effective repulsion between them. (2) Adding an attraction between the large and the small particles also results in an enhanced accumulation near the surface of the large particles and therefore in repulsive effective interactions. Furthermore, we found that for an attractive big-small interaction, adding smallsmall attractions resulted in even more effective repulsion. This "repulsion through attraction" effect is caused by a coupling between $\phi_{s s}(r)$ and $\phi_{b s}(r)$.

These predictions could in principle be verified in experiments that directly measure the effective forces of colloidal suspensions, such as optical tweezers $\| 7,58$ or Total Internal Reflection Microscopy (TIRM) 27,28,59. They could also be verified indirectly through measurements of phase-behavior and coagulation. Measurements of the second osmotic virial coefficient might also be very sensitive probes of the effective interactions 31. Systems where these interactions could be tuned include for example ternary suspensions 60] where the smallsmall attraction is generated by depletion attraction of an even smaller third colloidal component or highly salted charged suspensions where the van-der-Waals attraction dominates.

In some interesting recent experiments [61, a colloidal suspension of neutral big particles was stabilized by the addition of highly charged small nanoparticles. The proposed mechanism was termed "nanoparticle halos", and is very similar to the mechanism we observe, for example, in our run 2, where a repulsive $\phi_{s s}(r)$ of the screened Coulomb (Yukawa) form was added to the smaller particles, resulting in an increased accumulation of small particles near each big one, and an effective repulsion 
between the big particles. The effects in the experiments may also be enhanced by a small attractive $\phi_{b s}(r)$ (like our run 8). We are currently actively pursuing a more detailed comparison with these experiments.

Another possible application of this work is to supercritical solvents [62], which have important applications in industrial processes. The question of how the effective force on the big particles depends on the interactions with a low density solvent is encountered there as well 40,41, sometimes for similar size ratios.

More generally to make useful predictions for practical applications, one needs to more thoroughly explore the rather large parameter space, which includes the $\epsilon_{i j}$, the $\kappa_{i j}, \eta_{s}$ and the ratio $\sigma_{s s} / \sigma_{b b}$. This would be very tedious with simulations - trustworthy theoretical techniques would be more practical. We attempted a number of theoretical descriptions of the effective interactions. The superposition approximation, which has the advantage of only needing one-body input, works best for a strong big-small repulsion, but becomes much less accurate when the (local) small particle packing is high. When the smaller component is purely HS like, then our DFT approach is very accurate, just as was found for additive and non-additive HS mixtures [26, 30,31, 46]. When a repulsive interaction is added between the small particles, DFT does not perform quite as well, and when an attraction is added between the small particles, the differences are even more important. By mapping the small-small and big-small interactions onto effective HS diameters, we can map onto an effective non-additive HS model. This explains the qualitative trends for most of our parameter combinations. It is quantitative if only a small-small repulsion is added, but breaks down when both a small-small and a big-small interaction couple together to induce an enhanced small particle density.

All three tested theoretical methods perform less well for added small-small attractions. Constructing a reliable theory to treat this very interesting case is therefore a challenging problem. One possible way to extend the DFT calculations to an attractive small-small interaction would be to add a mean-field attractive term 55. For example, mean-field like functionals based on thermodynamic perturbation theory around a HS reference system have been successfully applied to the density profiles and phase-behavior in systems with attractive potentials [63,64.

New physics is expected when wetting or drying phenomena control the density of the small particles between the big ones. This is relevant if the bulk fluid of the small particles is close to liquid-gas phase coexistence. A wetting transition is expected to have a profound impact on the effective interaction as well, one important effect is a liquid "bridge" of small particles between the big one which has been recently studied in more detail 65].

We thank Jean-Pierre Hansen, Geoff Maitland, Edo Boek, and Bob Evans for illuminating discussions. AAL acknowledges support from the Isaac Newton Trust, Cambridge, HL acknowledges support from Schlum- berger Cambridge Research, through the Schlumberger visiting professor scheme.

[1] See e.g. W.B. Russel, D.A. Saville, and W.R. Schowalter, Colloidal Dispersions, Cambridge University Press, Cambridge (1989).

[2] P. N. Pusey, in Liquids, Freezing and the Glass Transition, edited by J. P. Hansen, D. Levesque and J. ZinnJustin (North Holland, Amsterdam, 1991)

[3] L. Belloni, J. Phys. Condens. Matter 12, R549 (2000).

[4] A.A. Louis, Phil. Trans. Roy. Soc. A 359, 939 (2001).

[5] C.N. Likos, Phys. Rep. 348, 267 (2001).

[6] E. Allahyarov and H. Löwen, J. Phys.: Condensed Matter 13, L277 (2001); Phys. Rev. E 63, 041403 (2001).

[7] J.C. Crocker, J. A. Matteo, A. D. Dinsmore, and A. G. Yodh, Phys. Rev. Lett. 82, 4352 (1999).

[8] S. Asakura and F. Oosawa, J. Chem. Phys. 22, 1255 (1954).

[9] S. Asakura and F. Oosawa, J. Polym. Sci., Polym. Symp. 33, 183 (1958); A. Vrij, Pure Appl. Chem. 48, 471 (1976).

[10] H.N.W. Lekkerkerker, W.C.K. Poon, P.N. Pusey, A. Stroobants and P.B. Warren, Europhys. Lett. 20, 559 (1992).

[11] S.M. Ilett, A. Orrock, W.C.K. Poon, and P.N. Pusey, Phys. Rev. E, 51, 1344 (1995).

[12] C.N. Likos, H. Löwen, M. Watzlawek, B. Abbas, O. Jucknischke, J. Allgaier, and D. Richter, Phys. Rev. Lett. 80 4450 (1998). M. Watzlawek, C.N. Likos, and H. Löwen, Phys. Rev. Lett. 82, 5289 (1999).

[13] A.A. Louis, P.G. Bolhuis, J.P. Hansen, and E.J. Meijer Phys. Rev. Lett. 852522 (2000).

[14] P.G. Bolhuis, A. A. Louis and J.P. Hansen, J. Chem. Phys. 114, 4296 (2001).

[15] P.G. Bolhuis, A. A. Louis, and J.P. Hansen, Phys. Rev. E.64, 021801 (2001).

[16] H. Löwen, J. P. Hansen, and P. A. Madden, J. Chem. Phys. 98, 3275 (1993).

[17] E. Allahyarov, I. D'Amico, and H. Löwen, Phys. Rev. Letters 81, 1334 (1998).

[18] E. Allahyarov, H. Löwen, and S. Trigger, Phys. Rev. E $\mathbf{5 7}, 5818$ (1998).

[19] In this hard-particle context non-additive means that the cross-diameter $\sigma_{b s} \neq \frac{1}{2}\left(\sigma_{s s}+\sigma_{b s}\right)$, where the $\sigma_{i j}$ are the HS diameters of the particles.

[20] T. Biben and J.P. Hansen, Phys. Rev. Lett. 66, 2215 (1991).

[21] T. Biben, P. Bladon, and D. Frenkel, J. Phys. Condensed Matter 8, 10799 (1996).

[22] R. Dickman, P. Attard, and V. Simonian, J. Chem. Phys. 107, 205 (1997).

[23] J.Y. Walz and A. Sharma J. Colloid Interface Sci. 168, 485 (1994).

[24] Y. Mao, M. E. Cates, and H. N. W. Lekkerkerker, Physica A 222, 10 (1995). 
[25] B. Götzelmann, R. Roth, S. Dietrich, M. Dijkstra, and R. Evans, Europhys. Lett. 47, 398 (1999).

[26] R. Roth, R. Evans, and S. Dietrich, Phys. Rev. E. 62, 5360 (2000).

[27] D. Rudhardt, C. Bechinger, and P. Leiderer, Phys. Rev. Lett. 81, 1330 (1998).

[28] C. Bechinger, D. Rudhardt, P. Leiderer, and R. Roth, S. Dietrich, Phys. Rev. Lett. 83, 3960 (1999).

[29] M. Dijkstra, R. van Roij and R. Evans, Phys. Rev. Lett. 81, 2268 (1998); ibid 82, 117 (1999); Phys. Rev. E. 59, 5744 (1999).

[30] A. A. Louis and R. Roth, J. Phys.: Condensed Matter 13, L777 (2001).

[31] R. Roth, R. Evans, and A.A. Louis to appear Phys. Rev. E 64, 051202 (2001)

[32] O. Mondain-Monval, F. Leal-Calderon, J. Philip, and J. Bibette, Phys. Rev. Lett. 75, 3364 (1995).

[33] R. Garibay-Alonso, J.M. Mendez-Alcaraz, and R. Klein, Physica A 235, 159 (1997).

[34] S. Amokrane, J. Chem. Phys. 108, 7459 (1998).

[35] J. Malherbe and S. Amokrane, Mol. Phys. 97, 677 (1999).

[36] H.H. von Grünberg and R. Klein, J. Chem. Phys. 110, 5421 (1999).

[37] J. Clément-Cottuz, S. Amokrane, and C. Regnaut, Phys. Rev. E 61, 1692 (2000).

[38] J.M. Méndez-Alcaraz and R. Klein, Phys. Rev. E. 61, 4095 (2000).

[39] M. Piech and J.Y. Walz, Langmuir 16, 7895 (2000).

[40] K. Kuwamoto and M. Kinoshita, Mol. Phys. 98, 725 (2000).

[41] S.A. Egorov and E. Rabani, J. Chem. Phys. 115, 617 (2001).

[42] S. Amokrane and J. G. Malherbe, J. Phys.: Condensed Matter 13, 7199 (2001).

[43] P. González-Mozuelos and J.M. Méndez-Alcaraz, Phys. Rev. E 63, 021201 (2001).

[44] P. Attard, J. Chem. Phys. 91, 3083 (1989).

[45] A.A. Louis, R. Finken and J.P. Hansen, Phys. Rev. E. 61, R1028 (2000).

[46] R. Roth and R. Evans, Europhys. Lett. 53, 271 (2001).

[47] W.G. McMillan and J.E. Mayer, J. Chem. Phys. 13, 276 (1945).

[48] Z. T. Nemeth and C. N. Likos, J. Phys.: Condensed Matter 7, L537 (1995).

[49] C. Caccamo, Phys. Rep. 274, 1 (1996).

[50] C. Caccamo, G. Pellicane, and D. Costa, J. Phys.: Condensed Matter 12, A437 (2000).

[51] H. Löwen and G, Kramposthuber, Europhys. Lett. 23 673 (1993).

[52] M. P. Allen, D. Tildesley, "Computer Simulation of Liquids", Oxford University Press, Oxford, 1991.

[53] E. Allahyarov and H. Löwen, Phys. Rev. E 63, 041403 (2001).

[54] J.P. Hansen and I.R. McDonald, Theory of Simple Liquids, 2nd Ed., Academic Press, London (1986).

[55] R. Evans, Adv. Phys. 28, 143 (1979).

[56] Y. Rosenfeld, Phys. Rev. Lett. 63, 980 (1989); J. Chem. Phys. 98, 8126 (1993).

[57] J.A. Barker and D. Henderson, J. Chem Phys. 47, 4714 (1967).
[58] R. Verma, J. C. Crocker, T. C. Lubensky, and A. G. Yodh Phys. Rev. Lett. 81, 4004 (1998).

[59] D.C. Prieve, Adv. Colloid Interface Sci. 82, 93 (1999).

[60] A. Konig and N.W. Ashcroft, Phys. Rev. E 63, 041203 (2001).

[61] V. Tohver, J.E. Smay, A. Braem, P.V. Braun, and J.A. Lewis, Proc. Natl. Acad. Sci. 98, 8950 (2001).

[62] Supercritical Fluids: Fundamentals for Applications, edited by E. Kiran and J. M. H. L. Sengers (Kluwer, Dordrecht, 1994).

[63] W. A. Curtin, N. W. Ashcroft, Phys. Rev. Lett. 56, 2775 (1986).

[64] R. Ohnesorge, H. Löwen, and H. Wagner, Phys. Rev. E 504801 (1994).

[65] C. Bauer, T. Bieker, and S. Dietrich, Phys. Rev. E 62, 5324 (2000).

TABLE I. Parameter combinations for simulation runs. The interactions are given by Eqs. (10) and (11), and only the $\epsilon_{b s}$ and $\epsilon_{s s}$ are changed; the other parameters are kept fixed.

\begin{tabular}{lcc}
\hline \hline run & $\beta \epsilon_{b s}$ & $\beta \epsilon_{s s}$ \\
\hline \hline 1 & 0 & 0 \\
2 & 0 & 2.99 \\
3 & 0 & -0.996 \\
\hline 4 & 0.82 & 0 \\
5 & 0.82 & 2.99 \\
6 & 0.82 & -0.996 \\
\hline 7 & -0.82 & 0 \\
8 & -0.82 & 2.99 \\
9 & -0.82 & -0.996 \\
\hline \hline
\end{tabular}




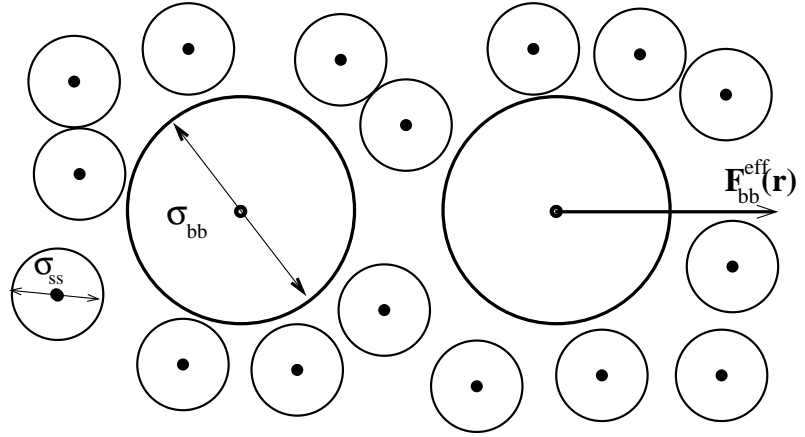

FIG. 1. Two big spheres of radius $\sigma_{b b}$ experience an effective force $F_{b b}^{e f f}(r)$ induced by the sea of small spheres or radius $\sigma_{s s}$.

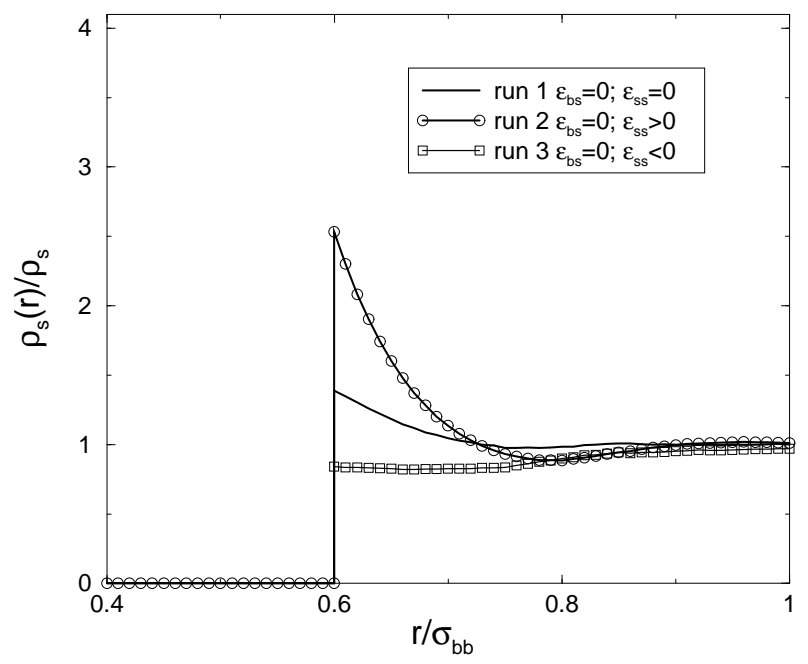

FIG. 2. Normalized density profiles $\rho_{s}(r) / \rho_{s}$ of centers of the small spheres as a function of the distance $r$ from the center of a single big sphere. Results are from computer simulations for runs $1-3$; i.e. $\epsilon_{b s}=0$, and $\epsilon_{s s}$ is varied. The values of the $\epsilon_{i j}$ can be found in table In this figure, as well as Figs. 3-6, the circles denote a repulsive $\epsilon_{s s}$, the squares an attractive $\epsilon_{s s}$ and no symbol means no added $\epsilon_{s s}$. Solid lines denote no added $\epsilon_{b s}$, dotted lines $\epsilon_{b s}>0$, and dot-dashed lines $\epsilon_{b s}<0$.

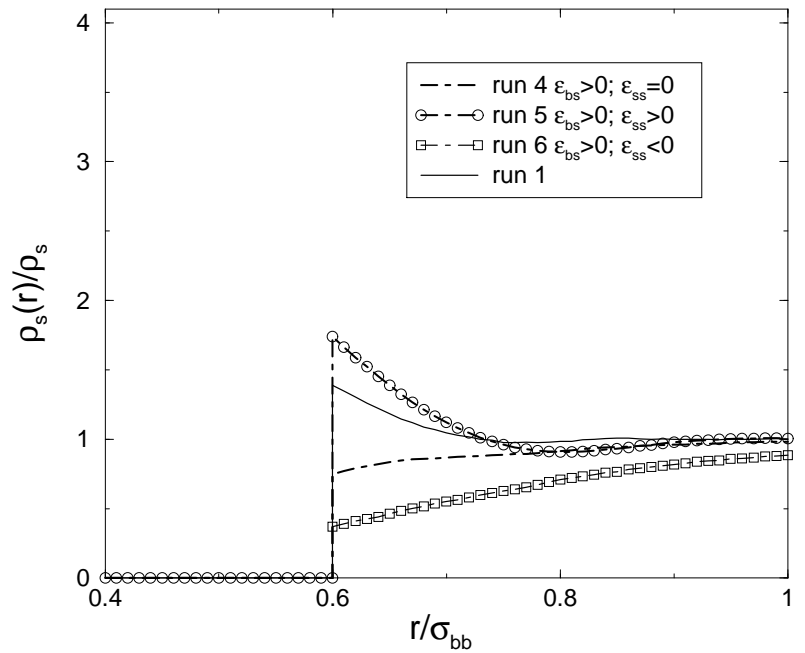

FIG. 3. Normalized density profiles $\rho_{s}(r) / \rho_{s}$ from computer simulations for runs $4-6 ; \epsilon_{b s}>0, \epsilon_{s s}$ is varied. We also show the result for run 1 , the pure HS case.

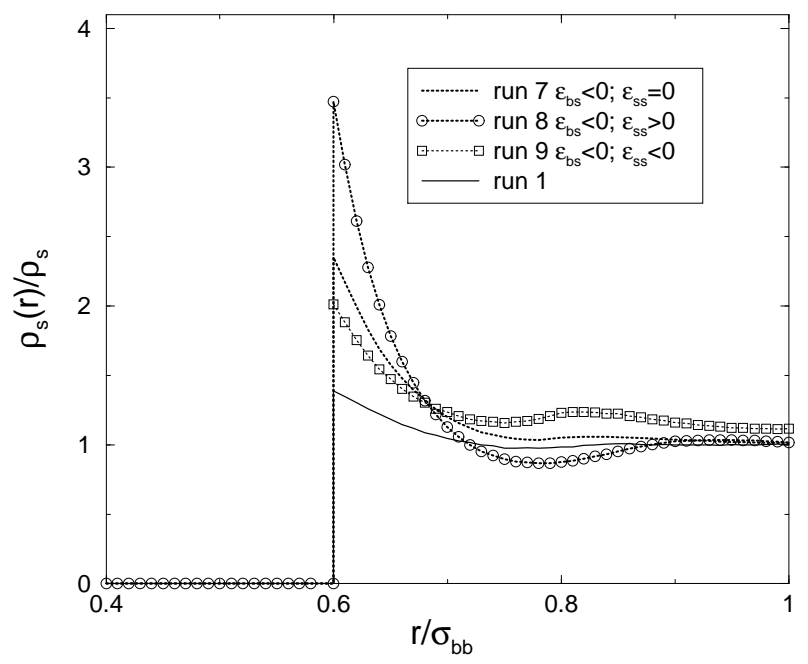

FIG. 4. Normalized density profiles $\rho_{s}(r) / \rho_{s}$ from computer simulations for runs $7-9 ; \epsilon_{b s}<0, \epsilon_{s s}$ is varied. We also show the result for run 1 , the pure HS case. 


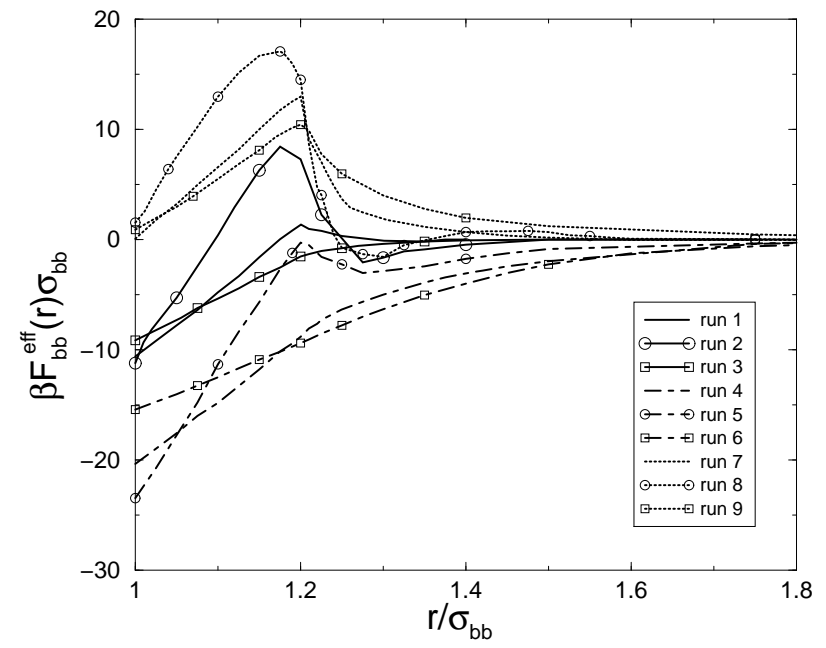

FIG. 5. Effective forces between the big particles, $\beta F_{b b}^{e f f}(r)$, shown for all 9 runs. The symbols are the same as in Figs. 2-4. The results are from direct computer simulations. Notice that the big-small interaction has the dominant effect. A repulsive $\epsilon_{b s}$ results in a more repulsive $\beta F_{b b}^{e f f}(r)$ (dot-dashed lines), while an attractive $\epsilon_{b s}$ results in a more attractive $\beta F_{b b}^{e f f}(r)$ (dotted lines). The small-small interactions have a relatively smaller effect.

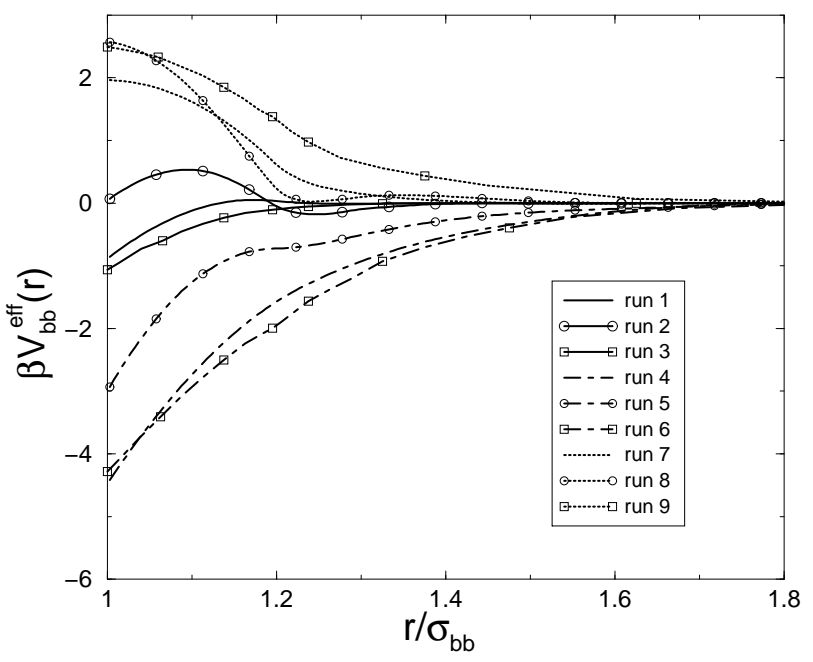

FIG. 6. Effective potentials between the big particles, $\beta V_{b b}^{e f f}(r)$, shown for all 9 runs. The results are from direct computer simulations. The symbols are the same as in Figs. 2-5.

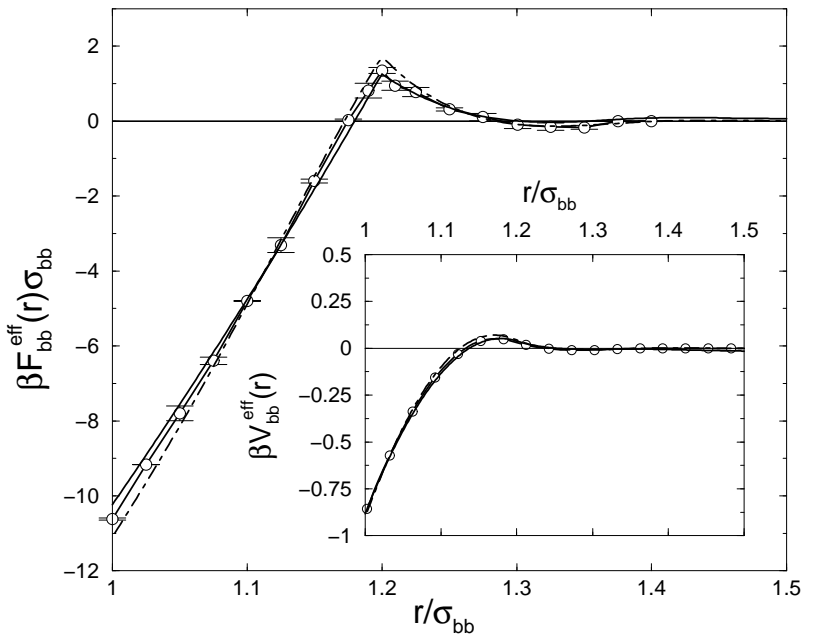

FIG. 7. Comparison of theory to simulation for run 1: $\epsilon_{b s}=0, \epsilon_{s s}=0$. Simulation (solid line with symbols), superposition approximation (solid line), and DFT (dot-dashed line) results for the effective force and potential (see inset) as a function of $r$, the distance between the centers of the big particles, are shown.

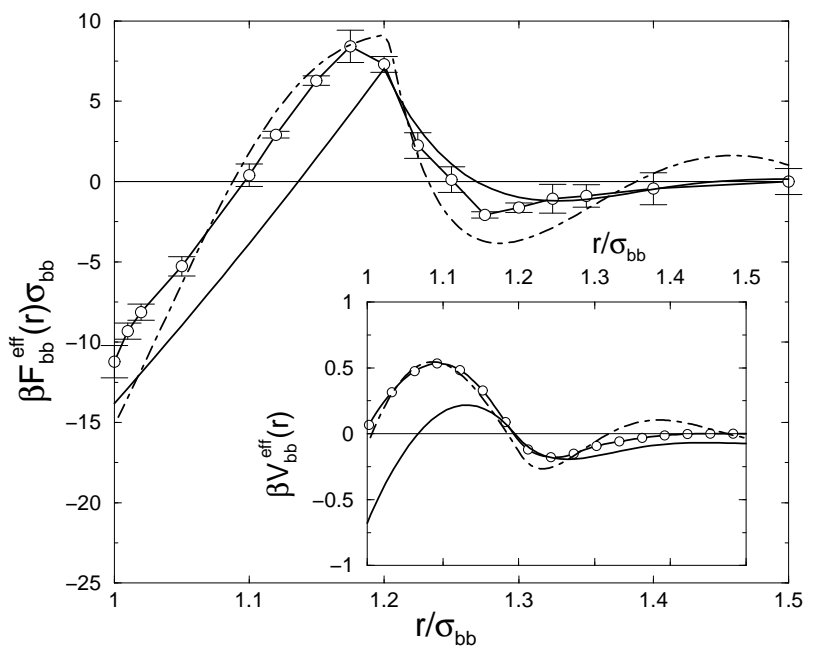

FIG. 8. Same as Fig. 7, but for run2: $\epsilon_{b s}=0, \epsilon_{s s}>0$. 


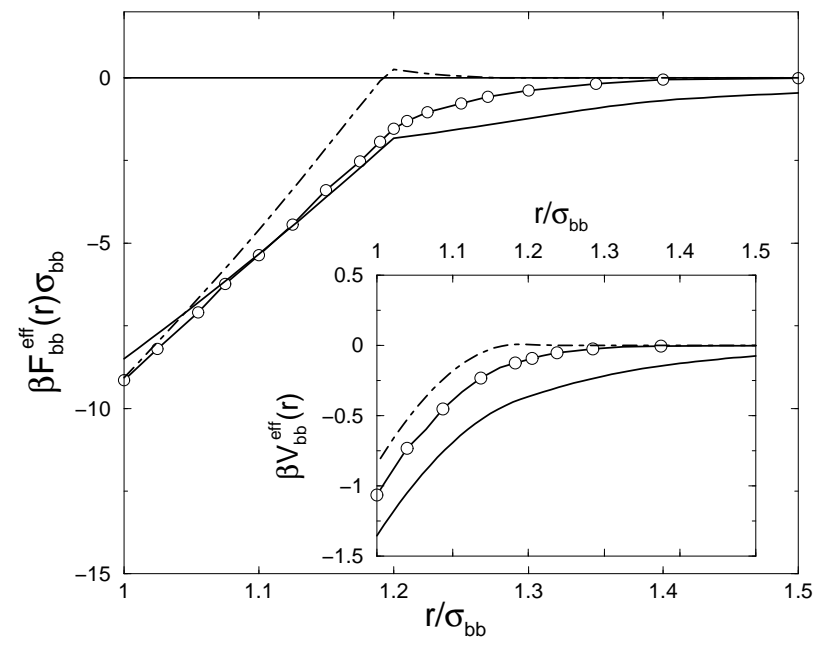

FIG. 9. Same as Fig. 17, but for run3: $\epsilon_{b s}=0, \epsilon_{s s}<0$.

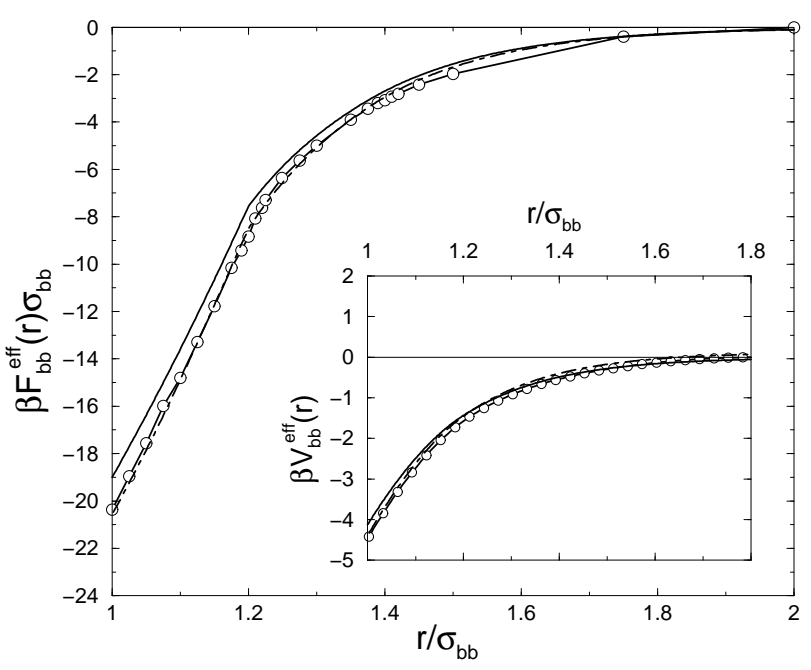

FIG. 10. Same as Fig. [, but for run4: $\epsilon_{b s}>0, \epsilon_{s s}=0$.

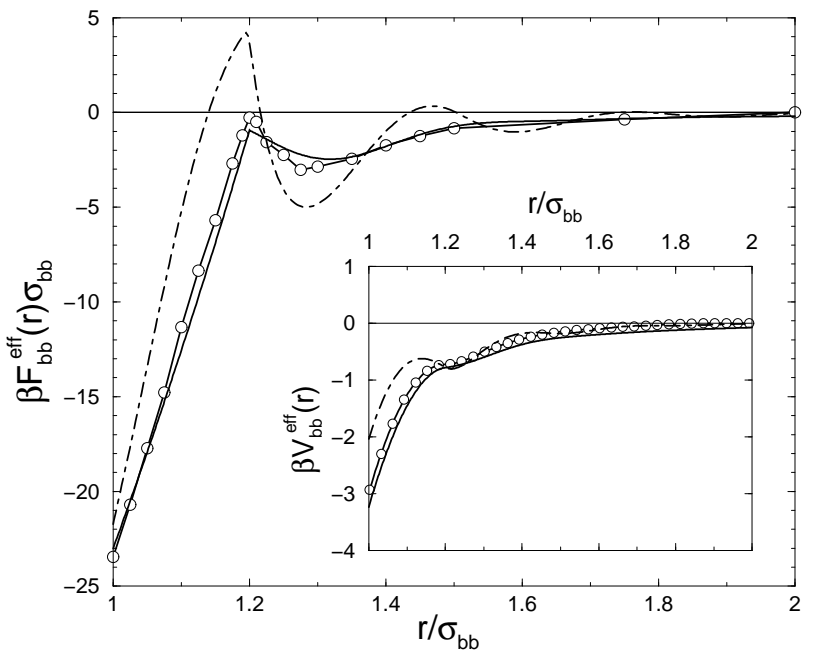

FIG. 11. Same as Fig. 母, but for run5: $\epsilon_{b s}>0, \epsilon_{s s}>0$.

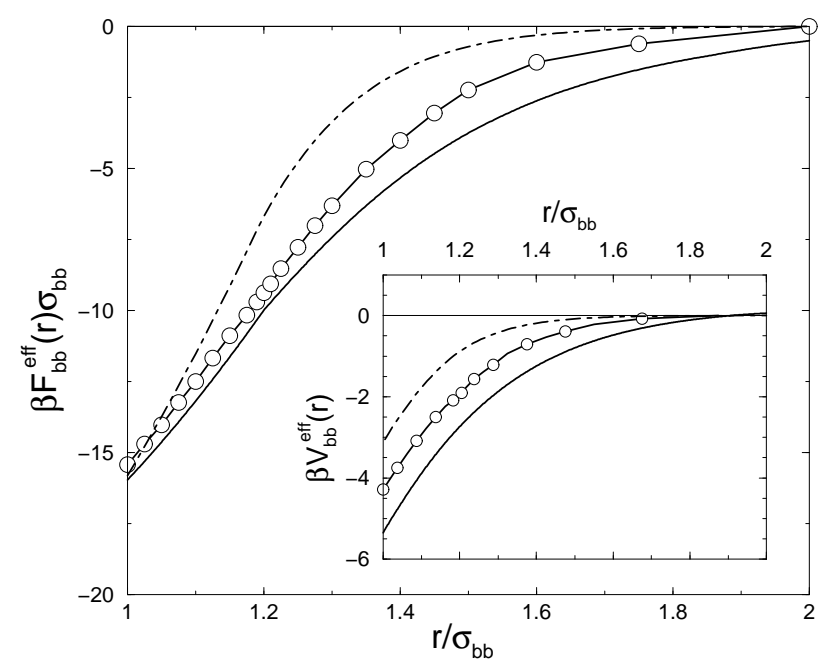

FIG. 12. Same as Fig. 月, but for run6: $\epsilon_{b s}>0, \epsilon_{s s}<0$.

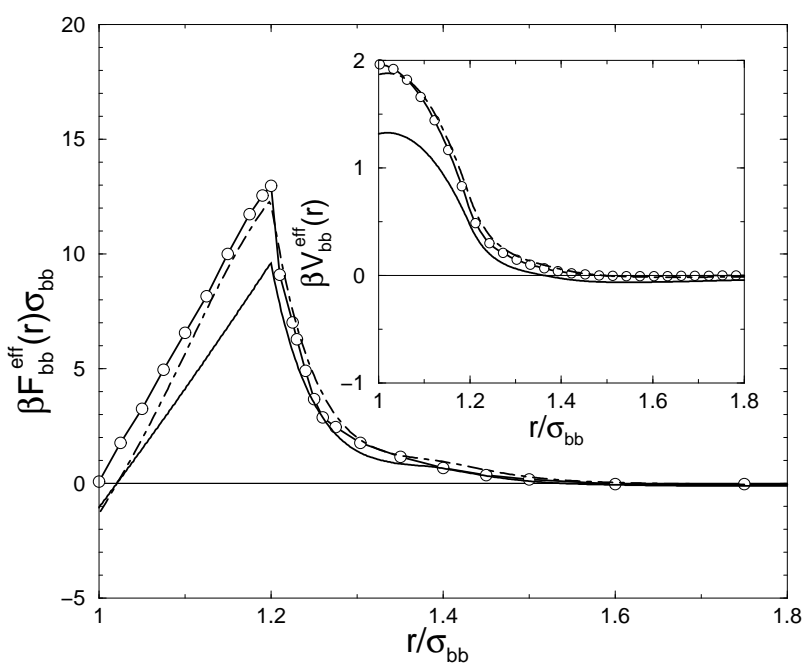

FIG. 13. Same as Fig. U, but for run7: $\epsilon_{b s}<0, \epsilon_{s s}=0$. 


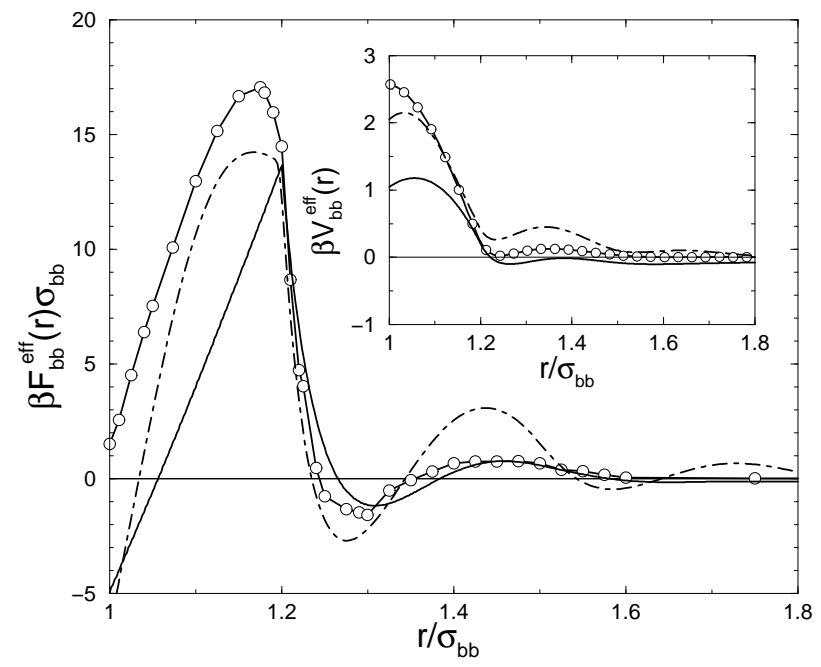

FIG. 14. Same as Fig. 月, but for run8: $\epsilon_{b s}<0, \epsilon_{s s}>0$. i

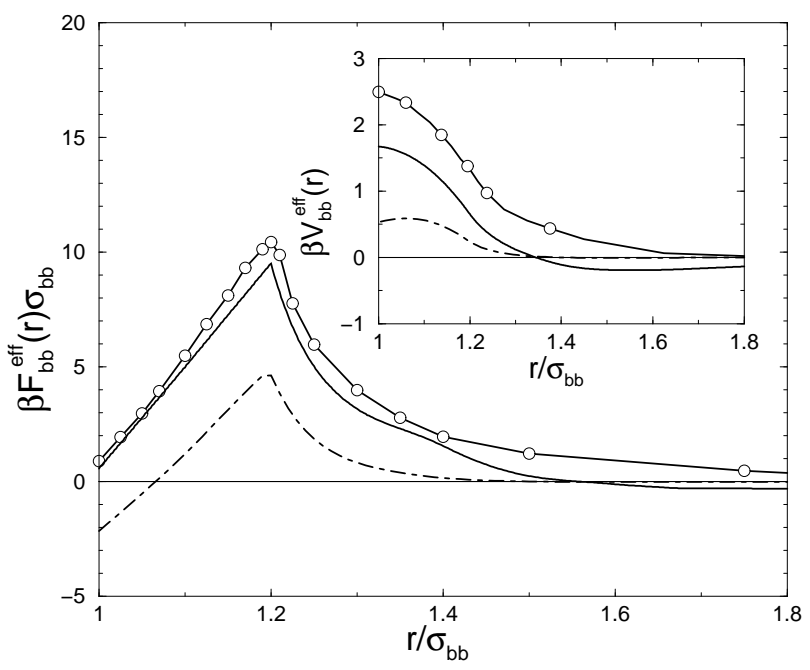

FIG. 15. Same as Fig. 月, but for run9: $\epsilon_{b s}<0, \epsilon_{s s}<0$. 